\title{
Dynamic Speckle Holography
}

\author{
S. Aime,${ }^{1,2, *}$ M. Sabato, ${ }^{1,3}$ L. Xiao, ${ }^{4, \dagger}$ and D.A. Weitz ${ }^{1,5}$ \\ ${ }^{1}$ John A. Paulson School of Engineering and Applied Sciences, \\ Harvard University, Cambridge, Massachusetts 02138, USA \\ ${ }^{2}$ Molecular, Macromolecular Chemistry, and Materials, ESPCI Paris, \\ CNRS, PSL University, 10 Rue Vauquelin, 75005 Paris, France \\ ${ }^{3}$ Department of Physics, The University of Chicago, Chicago, Illinois 60637, USA \\ ${ }^{4}$ State Key Laboratory of Petroleum Resources and Prospecting, \\ China University of Petroleum, Beijing, 102249, China \\ ${ }^{5}$ Department of Physics, Harvard University, Cambridge, Massachusetts 02138, USA
}

(Dated: August 23, 2021)

\begin{abstract}
We introduce Dynamic Speckle Holography (DSH), a new technique that combines imaging and scattering to measure three dimensional maps of displacements as small as ten nanometers over several centimeters, greatly extending the capabilities of traditional imaging systems. We attain this sensitivity by imaging speckle patterns of light collected at three scattering angles and measuring the decay in the temporal correlation due to local motion. We use DSH to measure the strain field of a colloidal gel undergoing fracture and establish the surprising role of internal tension in driving the fracture.
\end{abstract}

Measuring the dynamics of a system through the displacement of its constitutive elements is critical in many fields of research, from fluid dynamics to condensed matter physics. Such displacements are often measured by tracking features in images taken at different times: experimental methods such as particle tracking [1-9] or particle image velocimetry [10-13] are powerful and widelyused implementations of this method. However, these techniques all suffer from one key limitation: because they rely on tracking features in the image, their sensitivity, which is the smallest displacement they can probe, is intrinsically coupled to their resolution, which is the smallest size they can image. This precludes their use in applications involving small scale motion spread over large fields of view, such as occurs, for example, in fluidflow and mechanical instabilities [1, 14-18]. For instance, the propagation of a fracture entails fast, sub-micron motion extended over large distances $[16,19]$. This motion is extremely difficult to measure with real-time optical experiments, yet its detection is crucial to understand crack propagation in complex environments [20-25]. Similar limitations apply to many other phenomena, from turbulence, where flow is structured over many length scales from the system size to molecular scale [14], to plasticity and yielding, where mechanical energy is often dissipated by avalanche-like processes that are microscopic in nature but correlated over macroscopic distances [5, 18, 2630]. Measurement of motion at these microscopic length scales and over very large fields of view, using optical methods with the combination of spatial and temporal resolution, would enable investigation of many phenomena, for which our understanding has, so far, been hindered by the lack of experimental data.

In this paper we introduce Dynamic Speckle Holography (DSH), a novel imaging technique that detects displacements below the resolution limit set by diffraction, with a sensitivity completely independent of the resolution and the field of view. This is achieved by exploiting optical interference: DSH illuminates the sample using a coherent source, records the speckle pattern formed by the scattered light and analyzes the time evolution of each speckle to achieve sensitivity to displacements as small as $\sim 10 \mathrm{~nm}$. Using two lasers and two imaging detectors, DSH provides a full three-dimensional reconstruction of the displacement field with a spatial resolution of $\sim 100 \mu \mathrm{m}$ over a field of view spanning several centimeters. We validate DSH by investigating the flow of a Brownian suspension in a channel, showing that both Poiseuille flow and Brownian motion can be independently and simultaneously measured without resolving individual particles. In addition, we exploit DSH to reconstruct the complex deformation pattern surrounding the tip of a fracture propagating through a colloidal gel, and show that, unexpectedly, the gel pulls itself apart due to internal tension. Our results demonstrate the flexibility of DSH and illustrate its potential to investigate new phenomena.

We illustrate DSH by using it to study Poiseuille flow of a Brownian suspension in a channel. The sample is a $0.5 \mathrm{wt} \%$ suspension of 500-nm-diameter polystyrene particles in a 1:10 water-glycerol mixture, flowing in a channel with rectangular cross-section of $A=12.5 \times 0.12 \mathrm{~mm}^{2}$. The channel is illuminated by a laser beam, and a camera records images of the scattered light collected using a diaphragm and a lens, as shown in Fig. 1a. This geometry is similar to that used for Photon Correlation Imaging [31]: the sample is in the strictly single scattering limit and the camera collects only light scattered at an angle $\theta \approx 26^{\circ}$, corresponding to a scattering vector $\vec{q}$, with magnitude $q \approx 4.25 \mu \mathrm{m}^{-1}$, and oriented very close to the flow direction along the channel, as indicated in Fig. 1a. The optical setup has a large field of 
view, about $3 \times 2.4 \mathrm{~cm}^{2}$, but a low resolution, making it impossible to image individual particles. Instead, because of the laser coherence, images have a speckled appearance shown in Fig. 1b. Each speckle is the coherent superposition of light scattered by $\sim 10^{5}$ particles in a small volume of thickness $h=0.12 \mathrm{~mm}$ and lateral size $\sigma \approx 50 \mu \mathrm{m}$. By comparing images taken at two different times $t_{1}$ and $t_{2}$, we observe that the intensity of speckles generated by the flowing suspension fluctuates, whereas those generated by the channel walls remain constant, as shown in the insets of Fig. 1b. To quantify this temporal fluctuation, we compute the degree of correlation, $c_{I} \propto\left\langle I\left(t_{1}\right) I\left(t_{2}\right)\right\rangle-\left\langle I\left(t_{1}\right)\right\rangle\left\langle I\left(t_{2}\right)\right\rangle$, across the whole image, where $I$ is the intensity and the average is computed over groups of nearby pixels to retain the spatial resolution of the image while still providing a good measure of the temporal variation [32]. As the two speckle patterns become increasingly different, $c_{I}$ decays from 1 to 0 . For any pair of images, $c_{I}$ is homogeneous within the channel and depends only on time delay $\tau=t_{2}-t_{1}$. We average $c_{I}(\tau)$ from the fluid over space and time and find that, as the flow rate $Q$ is varied, the curves all have a similar shape, shown in Fig. 1c; each data set can be collapsed onto a single master curve when $\tau$ is rescaled by $v_{0}$, the flow speed in the middle of the channel, as shown in Fig. 1e. This provides a means of determining $v_{0}$ using DSH. Interestingly, each data set also exhibits clearly-defined damped oscillations, which remain clearly visible even upon scaling the data.

The dependence of $c_{I}(\tau)$ on $v_{0}$ stems from the dependence of the speckle intensity $I(t)$ on the phase of the light scattered by all particles, and therefore on their positions projected along the scattering vector $\vec{q}[33]$. Thus, relative motion of a fraction of wavelength in the direction of $\vec{q}$ results in fluctuation of $I(t)$, as captured by the decay of $c_{I}(\tau)$. Here, relative motion occurs because of the flow gradient imposed by the channel walls: particles in the middle of the channel flow at $v_{0}$, whereas particles close to the walls are almost immobile. Over a time delay $\tau$, they accumulate relative displacements as much as $v_{0} \tau$, which determines the decay of $c_{I}$ in Fig. 1e. The damped oscillations result from the alternating constructive and destructive interference between light scattered by the slowest and fastest particles contributing to each speckle [34]. We develop an analytical model to account for the parabolic velocity profile of Poiseuille flow and predict the correlation function (see Supplemental Material at [URL will be inserted by publisher] for the derivation, which includes Refs. [35-38]). The model fits the data very well, including the oscillations, as shown by the solid line in Fig. 1e. From the fit, we obtain a precise measure of $v_{0}$, in good agreement with the expected value $v_{e}=1.5 Q / A$, as shown in Fig. 1f. However, the model underestimates the damping of the oscillations for the slowest flow rates; this is because DSH is sensitive to all forms of motion, including the Brownian motion of the tracer particles, which becomes increasingly important as $Q$ decreases. We can generalize the model to include diffusive motion superposed to laminar flow: we use the diffusion coefficient $D=2.5 \cdot 10^{-3} \mu \mathrm{m}^{2} / \mathrm{s}$ computed using the Stokes-Einstein relation; the combined model captures the shape of the oscillations very well at all flow rates, as shown by the lines in Fig. 1c.

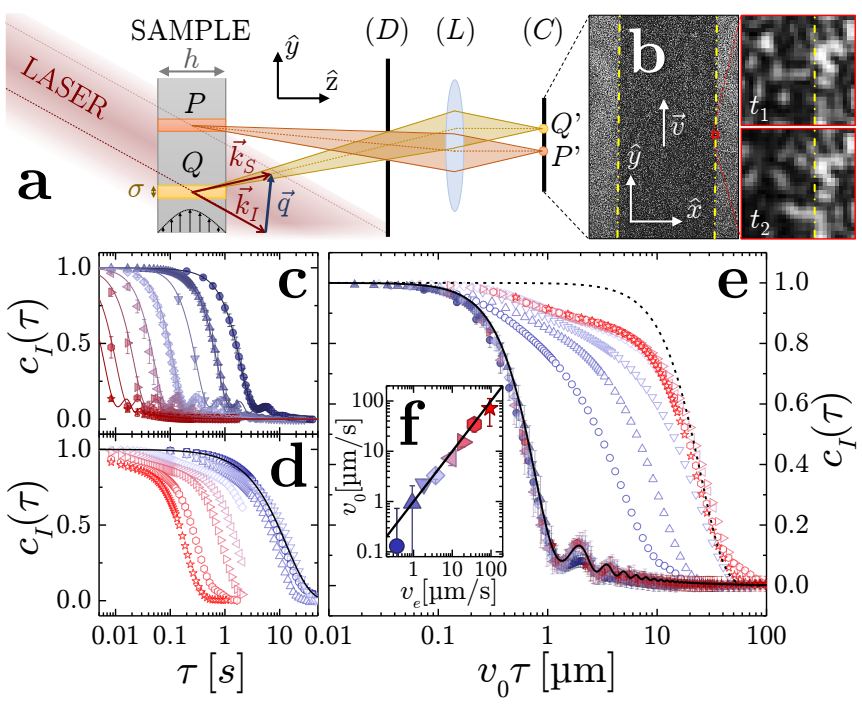

FIG. 1. (a) DSH geometry (not to scale): scattered light is collected by a camera $(C)$ through lens $(L)$ and diaphragm $(D)$, to image the speckles $\left(P^{\prime}, Q^{\prime}\right)$. (b) Speckle image of flow channel. Channel edges are highlighted by dashed lines. Insets: expanded region of $200 \times 200 \mu \mathrm{m}^{2}$, recorded at times $t_{1}$ and $t_{2}$. (c) Correlation functions measured with $\vec{q} \| \vec{v}$ for flow rate $Q=5,10,20,50,100,200,500,1000 \mathrm{~mm}^{3} / \mathrm{h}$ (full symbols, blue to red), all in the laminar flow regime. Lines: analytical model combining Poiseuille flow and Brownian motion. (d) Correlation functions measured with $\vec{q} \perp \vec{v}$, for same $Q$ (open symbols). Line: correlation decay due to Brownian motion. (e) Symbols: data from panels $(\mathrm{c}, \mathrm{d})$ plotted against $v_{0} \tau$. Solid line: correlation function for Poiseuille flow. Dashed line: correlation function for rigid speckle drift. (f) Poiseuille flow velocity $v_{0}$ as a function of expected value $v_{e}$. Line: $v_{0}=v_{e}$.

For the largest flow rates, the correlation functions decay faster than the acquisition rate of the camera. In this case, we can decrease the sensitivity of DSH to the flow by changing the orientation of the scattering vector relative to the flow direction: when $\vec{q}$ is perpendicular to the channel, the effect of flow on speckle fluctuation is reduced, and $c_{I}(\tau)$ exhibits a slower and qualitatively different decay, as shown in Fig. 1d. For the largest flow rates, the correlation functions again scale with $v_{0}$, collapsing onto a master curve when plotted as a function of $v_{0} \tau$, as shown in Fig. 1e. This decay is a result of speckles drifting rigidly in the flow direction at the average particle speed $v_{d}=2 v_{0} / 3$ : the correlation decays when the drift, $v_{d} \tau$, exceeds $\sigma$. Thus, $c_{I}$ can again be used to measure $v_{0}$, but here with a lower sensitivity, set by 
$\sigma$. In turn, $\sigma$ can be varied by changing the aperture of the diaphragm to investigate faster flow without increasing the data acquisition rate. This use of DSH is similar to laser speckle velocimetry (LSV) $[11,39]$. In contrast with LSV, however, DSH remains sensitive to both the motion of the speckles and to their fluctuation; this provides additional information about the microscopic dynamics. For the very smallest flow rates, speckle drift becomes negligible, and instead $c_{I}(\tau)$ exhibits an exponential decay, independent of $v_{0}$ : this is due to Brownian motion of the particles, as probed by traditional dynamic light scattering [33], and is represented by the solid line in Fig. 1d. These results illustrate the power of DSH to very sensitively probe different types of microscopic motion in three dimensions over a wide range of decay times and a large field of view.

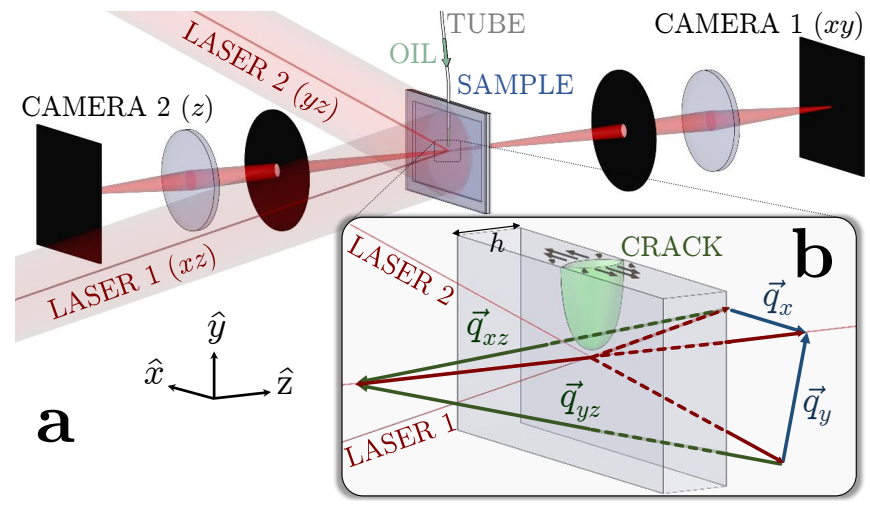

FIG. 2. (a) Optical configuration for three dimensional DSH. Two laser beams illuminate the sample, forming perpendicular scattering planes. Light scattered along the $\hat{z}$ axis is collected from both sides by mirrored optical setups composed of a diaphragm, a lens and a camera. (b) Orientation of the 4 scattering vectors formed by 2 incident wavevectors and 2 scattered wavevectors.

The real power of DSH is its ability to resolve complex, tiny motions in space and time over large areas. A good example of this type of motion is the elastic deformation field of a solid; this is intrinsically very small, yet correlated over a long range. We focus on the strain field around a propagating crack: this is particularly challenging because not only is the motion very small and over an extended range, but it is also non-stationary, evolving rapidly in time as the crack propagates. The sample is a colloidal gel formed by aggregating colloidal silica at a volume fraction of $2 \%$ by using urease to dissociate urea and increase the salt concentration to screen the stabilizing repulsive interaction between particles [40, 41]. The resultant gel has an elastic modulus of $\sim 1 \mathrm{kPa}$. To finely control the fracture propagation, we confine the gel between two transparent windows with a gap of $1 \mathrm{~mm}$, and inject mineral oil through a tube, as shown in Fig. 2a. As the fracture propagates, the speckle pattern fluctuates, indicating the gel is deforming. This is transient elastic deformation, in contrast to the stationary behavior of fluid flow. We therefore introduce a new way to analyze speckle dynamics that captures transient deterministic displacements of materials: we compute a correlation map $c_{I}\left(\vec{x} ; t_{1}, t_{2}\right)$ for every pair of frames, acquired at times $t_{1}$ and $t_{2}$, and use a grayscale, with darker shades corresponding to lower correlation, to show the spatial pattern of the motion. A typical correlation map exhibits darker regions around the inlet and the crack tip, as shown in Fig. 3a. The amount of decorrelation can be directly converted into the partial displacement $\Delta r\left(\vec{x} ; t_{1}, t_{2}\right)$ between $t_{1}$ and $t_{2}$, projected along the scattering vector $\vec{q}$. However, this conversion fails for $\Delta r \gtrsim 1 \mu \mathrm{m}$, as it results in complete loss of correlation. Similarly, $\Delta r \lesssim 100 \mathrm{~nm}$ have a large uncertainty because the loss of correlation is so small. Thus, to improve the measurement of both larger and smaller displacements, we compute $c_{I}$ for a given initial time $t_{1}$ and different final times $t_{2}$, and verify that the measured motion is additive: $\Delta r\left(t_{1}, t_{2}\right)+\Delta r\left(t_{2}, t_{3}\right)=\Delta r\left(t_{1}, t_{3}\right)$. We then integrate $\Delta r$ in time to resolve larger displacements and we differentiate it to improve the sensitivity to small motion. This enables reconstruction of displacements spanning nearly three orders of magnitude, between about $10 \mathrm{~nm}$ and several microns, as illustrated in the displacement map shown in Fig. 3b.

The displacement map $\Delta r\left(t_{1}, t_{2}\right)$ exhibits two lobes arranged horizontally in the $x$-direction, and a third one directly below them in the $y$-direction. While these are centered at the crack tip, they are not symmetric with the direction of crack propagation; they are instead symmetric with the scattering vector. This suggests that there may be a directional motion perpendicular to $\vec{q}$ that cannot be detected. To investigate this, we introduce a second laser beam such that the scattering vector points in the $y$ direction, as shown in Fig. 2. We alternate the illumination from the two laser beams, and collect images from the same propagating crack at the two perpendicular scattering vectors, $\vec{q}_{x}$ and $\vec{q}_{y}$. The displacement map along $\hat{y}$ exhibits a similar pattern as that along $\hat{x}$, but with $\hat{y}$ symmetry about the crack tip, as shown in Fig. 3c. In both cases, the measurement is sensitive only to the magnitude of the displacement, and not its direction. However, combining the two maps we obtain a two-dimensional displacement $\Delta \vec{r}$ that is symmetric about the crack and centered about the tip suggesting that motion is away from the crack. By integrating $\Delta \vec{r}$ from the beginning of the experiment to a given time $t$ we obtain the total displacements $\vec{R}(\vec{x}, t)$ accumulated since the fracture nucleation, which is directly related to the strain field, and can be used to gain insight into the behavior of the moving crack.

We find that $\vec{R}(\vec{x}, t)$ is largest close to the crack, and decays rapidly away from it, as shown by the arrows in Fig. 3e. Thus, we measure its magnitude $R$ as a function of the distance, $d$, from the crack, along the dashed line 
shown in Fig. 3e. For larger $d, R$ decays exponentially, as shown in Fig. 3f. This long range strain field corresponds to the elastic deformation induced by the pressure of the fluid driving crack propagation, assuming no-slip boundary conditions at the confining plates [42]. Under these conditions, the deformation of an ideal, incompressible material is similar to Poiseuille flow, with the maximum displacement halfway between the two confining plates, independent of $d$. By contrast, in a compressible material, $R$ decays exponentially with $d$, with a decay length determined by Poisson's ratio, $\nu$ [42]. A fit to the exponential tail of $R(d)$ yields $\nu=0.4996 \pm 10^{-4}$, indicating that the gel is nearly incompressible. Closer to the crack, $R$ deviates upwards: by subtracting the long range exponential contribution, we show that the excess in $R$ is well described by a second exponential decay, with a larger prefactor and a shorter decay length, as shown by the open symbols in Fig. 3f. This indicates that close to the crack the gel deforms more and can be more easily compressed.

To account for this excess deformation, we consider the consequences of the internal tension of the gel, which arises from the kinetics of its formation; since the gel is formed by cluster-cluster aggregation [43], two clusters will most likely stick to one another in a configuration that is not their equilibrated state, leading to an internal tension within the gel [44]. To validate the presence of internal tension we form the gel in a rheometer using parallel plate geometry and measure a negative normal force, pulling the plates towards each other during gelation, indicating an internal tension $\sigma_{z z}=-120 \mathrm{~Pa}$. Upon fracture, $\sigma_{z z}$ will pull the gel in all directions away from the fracture, both towards the intact gel and towards the confining windows; this results in a more complex deformation with motion in all three dimensions. We can use the power of DSH to directly measure this by also detecting motion perpendicular to the windows. We add a second optical setup, identical to the first, to collect backscattered light, as shown in Fig. 2a. This measures motion along two additional scattering vectors $\vec{q}_{x z}$ and $\vec{q}_{y z}$, with predominant components along the optical axis $\hat{u}_{z}$ and therefore sensitive to out-of-plane motion. Both $\vec{q}_{x z}$ and $\vec{q}_{y z}$ share the same in-plane components as their smallangle counterparts $\vec{q}_{x}$ and $\vec{q}_{y}$, as shown in Fig. 2b. Thus the speckle fields detected by both cameras are equally sensitive to in-plane motion, enabling us to isolate the contribution of out-of-plane motion. Relative out-ofplane displacements are qualitatively different from those in-plane: $c_{I}(\tau)$ decays exponentially, suggesting that part of the gel deforms in one direction while the other part deforms in the opposite direction. In this case, the correlation function depends on the mean square out-of-plane displacement $\Delta r_{z}^{2}$. This motion is enhanced close to the crack tip, and decays roughly isotropically away from it, as shown by the colormap in Fig. 3d. Again, $\Delta r_{z}^{2}$ is additive, so that $\Delta r_{z}^{2}\left(t_{1}, t_{2}\right)+\Delta r_{z}^{2}\left(t_{2}, t_{3}\right)=\Delta r_{z}^{2}\left(t_{1}, t_{3}\right)$ :

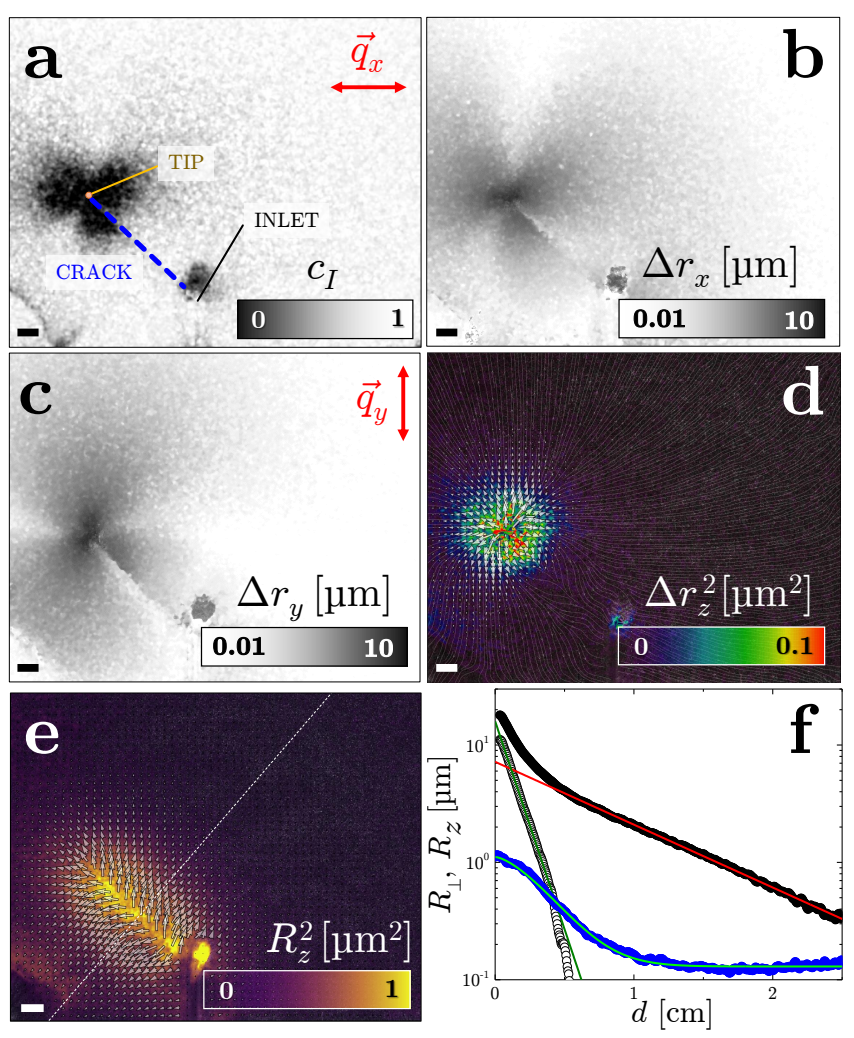

FIG. 3. (a) Correlation map from a propagating crack, indicated by the dashed blue line, measured with $\vec{q} \| \hat{x}$ for $\tau=1 \mathrm{~s}$. The scale bar corresponds to $1 \mathrm{~mm}$. (b-d) Maps of the partial displacements $\Delta \vec{r}$, accumulated over $\tau=1 \mathrm{~s}$. (b) Displacements along $\hat{x}$, represented with a logarithmic colormap; (c) Displacements along $\hat{y}$; (d) Combined map of displacements in the $x y$ plane (arrows) and along $\hat{z}$ (colormap). (e) Map of total displacements $\vec{R}$ relative to the sample at rest. (f) Deformation profile as a function of distance, $d$, from the crack. Full black symbols: in-plane displacements; red line: fit to the exponential tail; open black symbols: residuals of the exponential fit; blue symbols: out-of-plane displacements.

by integrating over time we obtain the total out-of-plane mean square displacement $R_{z}^{2}(\vec{x} ; t)$ accumulated through time $t$, which we show as a colormap in Fig. 3e. We find that $R_{z}^{2}$ is enhanced close to the fracture, and relaxes to a small plateau value with a characteristic length of $4 \mathrm{~mm}$. These results provide important insight about the nature of a crack propagating in a colloidal gel: surprisingly the internal tension pulls the gel apart as the crack propagates; this tension also pulls the gel towards the walls, resulting in motion in 3D. Further away from the fracture, the pressure of the driving fluid pushes the gel away from the crack. Here, the gel structure remains intact, and the motion is essentially planar and follows the exponential decay predicted by linear elasticity. These results highlight the power of DSH to reconstruct a rapidly evolving strain field surrounding the tip of the propagating crack, thus measuring very small motion over a large area. 
Dynamic speckle holography is a powerful imaging technique that relies on interference of scattered light rather than the resolution of imaged light, enabling very small three-dimensional motions to be measured over large fields of view. Moreover, DSH can be applied to non-stationary motion such as the strain field induced by fracture or other stochastic displacements such as those involved in plasticity. Its sensitivity makes DSH an ideal technique to probe motion that is microscopic yet structured over large length scales, such as turbulent flow or collective dynamics in active or externally driven systems.

We thank Jun Yin for help with experiments and Shmuel Rubinstein for insightful discussions. This work was supported by CUPB (CUP grant B13010), NSF Award DMR-1708729 and through the Harvard University Materials Research Science and Engineering Center (NSF Grants DMR-1420570 and DMR-2011754).

* stefano.aime@espci.fr

$\dagger$ †xiaolizhi@cup.edu.cn

[1] P. E. Arratia, C. C. Thomas, J. Diorio, and J. P. Gollub, Physical Review Letters 96, 144502 (2006).

[2] T. G. Mason, K. Ganesan, J. H. van Zanten, D. Wirtz, and S. C. Kuo, Physical Review Letters 79, 3282 (1997).

[3] R. Besseling, L. Isa, E. R. Weeks, and W. C. K. Poon, Advances in Colloid and Interface Science 146, 1 (2009).

[4] M. Bierbaum, B. D. Leahy, A. A. Alemi, I. Cohen, and J. P. Sethna, Physical Review X 7, 041007 (2017).

[5] P. Schall, D. A. Weitz, and F. Spaepen, Science 318, 1895 (2007).

[6] C. Manzo and M. F. Garcia-Parajo, Reports on Progress in Physics 78, 124601 (2015).

[7] T. G. Dimiduk and V. N. Manoharan, Optics Express 24, 24045 (2016), publisher: Optical Society of America.

[8] M. Tennenbaum, Z. Liu, D. Hu, and A. FernandezNieves, Nature Materials 15, 54 (2016), number: 1 Publisher: Nature Publishing Group.

[9] A. Malloy and B. Carr, Particle and Particle Systems Characterization 23, 197 (2006).

[10] D. H. Barnhart, R. J. Adrian, and G. C. Papen, Applied Optics 33, 7159 (1994), publisher: Optical Society of America.

[11] R. J. Adrian, Experiments in Fluids 39, 159 (2005).

[12] P. T. Tokumaru and P. E. Dimotakis, Experiments in Fluids 19, 1 (1995).

[13] E. J. Stamhuis and J. J. Videler, The Journal of Experimental Biology 198, 283 (1995).

[14] R. McKeown, R. Ostilla-Mónico, A. Pumir, M. P. Brenner, and S. M. Rubinstein, Science Advances 6, eaaz2717 (2020), publisher: American Association for the Advancement of Science Section: Research Article.

[15] A. Groisman and V. Steinberg, Nature 405, 53 (2000), number: 6782 Publisher: Nature Publishing Group.
[16] J. Fineberg and M. Marder, Physics Reports , 108 (1999).

[17] A. Livne, E. Bouchbinder, I. Svetlizky, and J. Fineberg, Science 327, 1359 (2010).

[18] A. Le Bouil, A. Amon, S. McNamara, and J. Crassous, Physical Review Letters 112, 10.1103/PhysRevLett.112.246001 (2014).

[19] C. Creton and M. Ciccotti, Reports on Progress in Physics 79, 046601 (2016).

[20] T. G. Boue, G. Cohen, and J. Fineberg, Physical Review Letters 114, 054301 (2015).

[21] B. Cotterell and J. Rice, International Journal of Fracture 16, 155 (1980).

[22] M.-m. Schwaab, T. Biben, S. Santucci, A. Gravouil, and L. Vanel, Physical Review Letters 120, 255501 (2018).

[23] S. P. Morgan, C. A. Johnson, and H. H. Einstein, International Journal of Fracture 180, 177 (2013).

[24] K. H. Nam, I. H. Park, and S. H. Ko, Nature 485, 221 (2012).

[25] T. Baumberger and O. Ronsin, The European Physical Journal E 31, 51 (2010).

[26] J. P. S. Christopher R. Myers, Nature 410, 242 (2001).

[27] A. Nicolas, E. E. Ferrero, K. Martens, and J.-L. Barrat, Reviews of Modern Physics 90, 045006 (2018).

[28] P.-A. Lemieux and D. J. Durian, Physical Review Letters 85, 4273 (2000), arXiv: cond-mat/0005388.

[29] T. Sentjabrskaja, P. Chaudhuri, M. Hermes, W. C. K. Poon, J. Horbach, S. U. Egelhaaf, and M. Laurati, Scientific Reports 5, 11884 (2015).

[30] S. Jamali, R. C. Armstrong, and G. H. McKinley, Physical Review Letters 123, 248003 (2019).

[31] A. Duri, D. A. Sessoms, V. Trappe, and L. Cipelletti, Physical Review Letters 102, 085702 (2009).

[32] L. Cipelletti, H. Bissig, V. Trappe, P. Ballesta, and S. Mazoyer, Journal of Physics: Condensed Matter 15, S257 (2002), publisher: IOP Publishing.

[33] B. J. B. Robert Pecora, Dynamic Light Scattering with Applications to Chemistry, Biology, and Physics (John Wiley and Sons).

[34] S. Aime and L. Cipelletti, Soft Matter 15, 200 (2019).

[35] J. W. Goodman, Introduction to Fourier Optics (McGraw-Hill).

[36] S. Aime, DSHpy (2020), GitHub repository, https:// github.com/steaime/DSHpy.

[37] R. Cerbino and V. Trappe, Physical Review Letters 100 (2008-05-05).

[38] S. Aime and L. Cipelletti, Soft Matter 15, 213 (2019).

[39] M. D. Alaimo, D. Magatti, F. Ferri, and M. A. C. Potenza, Applied Physics Letters 88, 191101 (2006).

[40] S. Aime, L. Cipelletti, and L. Ramos, Journal of Rheology 62, 1429 (2018).

[41] S. Aime, L. Ramos, and L. Cipelletti, Proceedings of the National Academy of Sciences , 201717403 (2018).

[42] S. Timoshenko and J. Goodier, Theory of Elasticity (McGraw-Hill, 1951).

[43] S. Manley, B. Davidovitch, N. R. Davies, L. Cipelletti, A. E. Bailey, R. J. Christianson, U. Gasser, V. Prasad, P. N. Segre, M. P. Doherty, S. Sankaran, A. L. Jankovsky, B. Shiley, J. Bowen, J. Eggers, C. Kurta, T. Lorik, and D. A. Weitz, Physical Review Letters 95, 048302 (2005).

[44] M. Bouzid and E. Del Gado, Langmuir 34, 773 (2018). 